\title{
Extravasation during bladder cancer metastasis requires cortactin-mediated invadopodia formation
}

\author{
NORIKO TOKUI ${ }^{1}$, MIHOKO SUTOH YONEYAMA ${ }^{1,2}$, SHINGO HATAKEYAMA $^{1}$, \\ HAYATO YAMAMOTO $^{1}$, TAKUYA KOIE ${ }^{1}$, HISAO SAITOH ${ }^{3}$, KANEMITSU YAMAYA ${ }^{3}$, \\ TOMIHISA FUNYU ${ }^{3}$, TOSHIYA NAKAMURA ${ }^{4}$, CHIKARA OHYAMA $^{1}$ and SHIGERU TSUBOI ${ }^{1,2}$ \\ ${ }^{1}$ Department of Urology, Hirosaki University Graduate School of Medicine, Hirosaki, \\ Aomori 036-8562; Departments of ${ }^{2}$ Cancer Immunology and Cell Biology and ${ }^{3}$ Urology, \\ Oyokyo Kidney Research Institute, Hirosaki, Aomori 036-8243; ${ }^{4}$ Department of Biomedical Sciences, \\ Hirosaki University Graduate School of Health Sciences, Hirosaki, Aomori 036-8564, Japan
}

Received July 17, 2013; Accepted February 2, 2014

DOI: $10.3892 / \mathrm{mmr} .2014 .1965$

\begin{abstract}
Invasive cancer cells form the filamentous actin-based membrane protrusions known as invadopodia. Invadopodia are thought to play a critical role in cancer cell invasion and metastasis due to their ability to degrade the extracellular matrix. The present study assessed whether invadopodia formation is essential in extravasation of circulating bladder cancer cells and lung metastasis. To analyze the importance of invadopodia, bladder cancer cell lines with reduced invadopodia formation were established by silencing the expression of cortactin, an essential component of invadopodia, using cortactin short hairpin RNA. Bladder cancer cells with cortactin knockdown demonstrated a markedly decreased ability to form invadopodia, secrete matrix metalloproteinases and invade the extracellular matrix. In addition, the knockdown cells exhibited a reduced transendothelial invasion capacity and decreased formation of metastatic foci in the lungs. The present study demonstrated that bladder cancer cells with cortactin knockdown have a reduced capacity to extravasate into the lung from the circulation, due to the decreased invasive character of invadopodia. This suggests that invadopodia formation is a critical process for cancer cell extravasation.
\end{abstract}

\section{Introduction}

The majority of cancer mortalities are caused by metastasis. Hematogenous metastasis includes multiple steps. First, invasive cancer cells escape from the primary site into nearby blood vessels (intravasation) and disseminate around the body through the host circulation. Following this, the circulating cancer cells

Correspondence to: Dr Shigeru Tsuboi, Department of Urology, Hirosaki University School of Medicine, 5 Zaifu-cho, Hirosaki, Aomori 036-8562, Japan

E-mail: urology@cc.hirosaki-u.ac.jp

Key words: bladder cancer, metastasis, extravasation, invadopodia, cortactin exit the vessels to cross the endothelia and invade secondary organ tissue (extravasation), where they proliferate to form metastases (1). It is vital to elucidate the detailed processes of these steps to prevent metastasis, however, at present, the molecular mechanisms underlying each step remain poorly understood.

In order to invade surrounding tissues, invasive cancer cells form invadopodia, the filamentous actin (F-actin)-based membrane protrusions required for degradation of the extracellular matrix (ECM) and migration through the tissues (2-4). It has been reported that invadopodia play an essential role in the intravasation of cancer cells from the primary site into the blood vessels (5). However, the importance of invadopodia formation in extravasation from the blood vessels into the target organs during metastasis, remains poorly understood.

In patients with aggressive bladder cancer, the most common site of hematogenous metastasis is the lungs (6). The circulating bladder cancer cells exit the lung microvessels by transendothelial invasion, to enter the lung tissue. The present study assessed whether invadopodia formation is involved in extravasation by analyzing lung metastasis of aggressive bladder cancer cells.

\section{Materials and methods}

Cells, reagents and antibodies. A human invasive and high-grade bladder cancer cell line, YTS-1, was provided by Dr H. Kakizaki (Yamagata University, Yamagata, Japan). YTS-1 cells were maintained in RPMI-1640 medium (Sigma-Aldrich, St. Louis, MO, USA) supplemented with $10 \%$ fetal bovine serum (FBS; PAA Laboratories $\mathrm{GmbH}$, Pasching, Austria) with $5 \% \mathrm{CO}_{2}$ at $37^{\circ} \mathrm{C}$. Primary human lung microvascular endothelial cells (HMVEC-L) were purchased from Lonza (Walkersville, MD, USA) and maintained in EGM-2MV medium (Lonza). All biochemical reagents were purchased from Sigma-Aldrich unless otherwise noted. Anti-cortactin monoclonal antibody (clone, EP1922Y) and anti-actin polyclonal antibody were purchased from Epitomics Inc. (Burlingame, CA, USA) and Sigma-Aldrich, respectively.

Stable transfectants. YTS-1 cells with reduced expression of cortactin were generated by short hairpin (sh) RNA technology 
as previously described (7). An shRNA expression plasmid was constructed using pBAsi-hU6 Neo DNA (Takara Bio, Inc., Shiga, Japan). The shRNA sequence for cortactin was: GATC CGCACGAGTCACAGAGAGATCTGTGAAGCCACAGATG GGATCTCTCTGTGACTCGTGCT TTTTTA, the small interfering (si) RNA sequence for cortactin is underlined. A human non-targeting siRNA sequence (Accell Control siRNA kit, Thermo Fisher Scientific, Waltham, MA, USA) was used to prepare the control cells expressing non-targeting shRNA. The shRNA expression plasmids (knockdown and control constructs), together with pTK-HyB, were introduced into YTS-1 cells in a 10:1 molar ratio using Lipofectamine 2000 (Invitrogen Life Technologies, Carlsbad, CA, USA). Drug-resistant colonies were selected in the presence of $200 \mu \mathrm{g} / \mathrm{ml}$ hygromycin B. Two knockdown clones (designated cortKD-1 and -2) were selected based on the cortactin expression levels. cortKD-1 and -2, and one control clone (designated YTS control), were used for the assays described in this study.

Western blot analysis. Total lysates of cancer cells were prepared by solubilization in $50 \mathrm{mM}$ Tris- $\mathrm{HCl}$ buffer $(\mathrm{pH} 7.5)$ containing $1 \%$ Igepal CA-630, $150 \mathrm{mM} \mathrm{NaCl}$ and proteinase inhibitors. The lysates were resolved by SDS-PAGE on an $8-16 \%$ gradient gel (Invitrogen Life Technologies) and transferred to polyvinylidene fluoride membrane. Western blotting was performed using specific primary antibodies and a horseradish peroxidase-conjugated secondary antibody. Signals were visualized using the ECL PLUS detection system (GE Healthcare, Amersham, UK).

Immunofluorescence microscopy. Cells seeded on coverslips were fixed in $4 \%$ paraformaldehyde and permeabilized with phosphate-buffered serum (PBS) containing $0.1 \%$ saponin and $1 \%$ bovine serum albumin. Cells were stained with Alexa Fluor 568-labeled phalloidin (Invitrogen Life Technologies), together with the monoclonal antibody against cortactin, and Alexa Fluor 488-labeled secondary antibody (Invitrogen Life Technologies) was used for primary antibody detection. Cell staining was examined under an Olympus IX-71 fluorescence microscope (Olympus Inc., Tokyo, Japan) and LSM 710 Laser Scanning confocal microscope (Carl Zeiss, Oberkochen, Germany).

Gelatin zymography. Gelatin zymography was performed in a $10 \%$ Novex Zymogram pre-cast SDS-PAGE gel (Invitrogen Life Technologies) in the presence of $0.1 \%$ gelatin under nonreduced conditions. Cells $\left(1 \times 10^{6}\right)$ in $2 \mathrm{ml}$ RPMI-1640 containing $10 \%$ FBS were placed in a single well (6-well dish) and grown to $80 \%$ confluence. Cells were washed with PBS and incubated with $2 \mathrm{ml}$ Opti-MEM (Invitrogen Life Technologies) for $24 \mathrm{~h}$. Conditioned media were collected and subjected to SDS-PAGE. Gels were washed in $2.5 \%$ Triton $\mathrm{X}-100$ for $30 \mathrm{~min}$ at room temperature to remove SDS and incubated at $37^{\circ} \mathrm{C}$ overnight in substrate buffer containing $50 \mathrm{mM}$ Tris- $\mathrm{HCl}\left(\mathrm{pH} 8.0\right.$ ) and $5 \mathrm{mM} \mathrm{CaCl}_{2}$. Following this, cells were stained with $0.5 \%$ Coomasie Brilliant Blue R-250 in 50\% methanol and $10 \%$ acetic acid for $1 \mathrm{~h}$. The bands of matrix metalloprotease-2 (MMP-2) appear as clear bands against dark background due to its gelatin degradation activity.
Matrigel and transendothelial invasion assays. The two invasion assays were performed using a conventional Transwell system (BD Biosciences, San Jose, CA, USA). For the Matrigel invasion assay, the bottom surface of the upper chamber filter (8- $\mu \mathrm{m}$ pore size) was coated with $100 \mu \mathrm{g} / \mathrm{ml}$ fibronectin and the top surface was covered with $1 \mathrm{mg} / \mathrm{ml}$ Matrigel matrix (BD Biosciences). The lower chamber was filled with serum-free RPMI-1640 medium. Bladder cancer cells $\left(5 \times 10^{4}\right)$ were labeled with the Vybrant CFDA-SE Cell Tracer kit (Invitrogen Life Technologies) and placed in the upper chamber. Following incubation at $37^{\circ} \mathrm{C}$ for $24 \mathrm{~h}$, non-migrated cells remaining at the top surface of the filter were carefully removed with cotton swabs. Migrated cells on the bottom surface were fixed with $4 \%$ paraformaldehyde and counted under a fluorescence microscope (Olympus IX-71). For transendothelial invasion assay, HMVEC-L cells $\left(1 \times 10^{5}\right)$ were placed onto a collagen I-coated upper chamber $(8-\mu \mathrm{m}$ pore size) and cultured over 2 days to form a monolayer. Carboxyfluorescein diacetate-succinimidyl ester-labeled bladder cancer cells were re-suspended in EGM-2MV medium and cells $\left(5 \times 10^{4}\right)$ were added onto the HMVEC-L monolayer. Following incubation at $37^{\circ} \mathrm{C}$ for $24 \mathrm{~h}$, non-migrated cells were removed and migrated cells were fixed and counted.

Lung metastasis assay in nude mice. Bladder cancer cells $\left(2 \times 10^{6}\right)$ were suspended in $0.1 \mathrm{ml}$ serum-free RPMI-1640 medium and injected into the tail vein of 6- to 8-week-old nude mice (BALB/cAJcI-nu/nu; Clea Japan, Inc., Tokyo, Japan). After 3 weeks, lungs were harvested and fixed with formalin. The left lung was removed and trisected. The lung blocks were cut further into 4- $\mu \mathrm{m}$ sections and stained with hematoxylin and eosin (HE). Lung metastatic foci were identified by $\mathrm{HE}$ staining based on the histopathological findings, for example abnormally high cell density and atypical nuclear morphology. The degree of metastasis was evaluated by the proportion (\%) of the tumor area occupying the total lung area of the section. Images of the lung were captured with a scanner (Epson Perfection 4990; Epson, Tokyo, Japan) and the proportion (\%) of the metastasized tumor area was calculated using DP2-BSW software (Olympus). The experiments were approved by the committee for animal experiments of Oyokyo Kidney Research Institute (Hirosaki Hospital, Hirosaki, Japan).

Statistical analysis. The SPSS 12.0 statistical program (SPSS, Chicago, IL, USA) was used. Statistically significant differences were determined using the Student's t-test. $\mathrm{P}<0.05$ was considered to indicate a statistically significant difference.

\section{Results}

Establishment of cortactin knockdown bladder cancer cells. To determine the contribution of invadopodia formation to extravasation, the effect of a reduction of invadopodia formation on cancer cell extravasation was analyzed. To reduce invadopodia formation, the expression of cortactin in invasive bladder cancer cells was silenced, as cortactin is an invadopodium marker and one of the most important regulators for invadopodia formation. Several stable cortactin knockdown cell lines were established using an invasive bladder cancer cell line, YTS-1. A YTS-1 cell line expressing non-targeting 

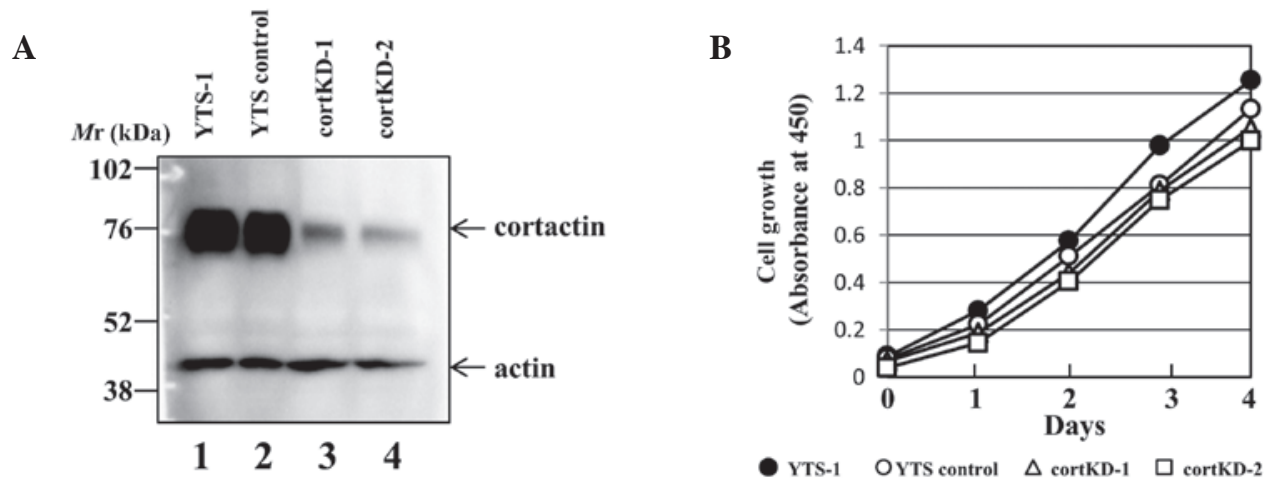

Figure 1. Establishment of cortactin knockdown bladder cancer cells. (A) Expression levels of cortactin were reduced in cortactin knockdown cells. Total protein samples prepared form YTS-1 parent, YTS control, cortKD-1 and corKD-2 cells were analyzed by western blotting for the expression of cortactin and actin. (B) Cell growth of the YTS-derived cells. Growth kinetics of YTS-1 (parent cell), YTS control, cortKD-1 and cortKD-2 cells were measured using a Cell Counting kit-8, based on their cellular dehydrogenase activity. Results represent one of three independent experiments. Cort, cortactin; KD, knockdown.

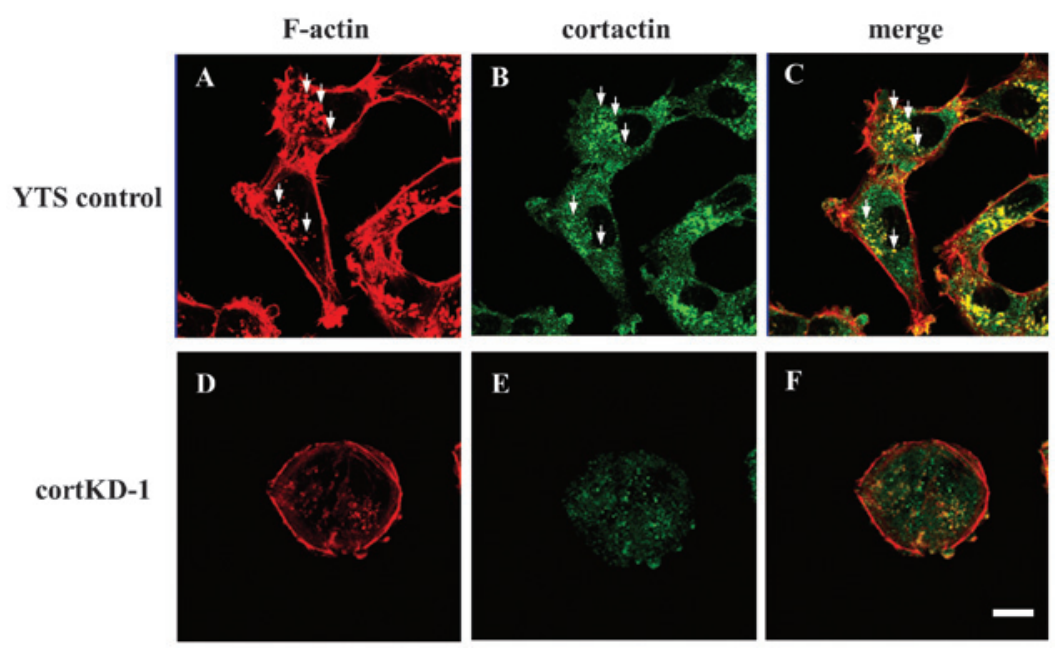

Figure 2. Reduced invadopodia formation in cortactin knockdown bladder cancer cells. Cells were double-stained with Alexa Fluor 568-labeled phalloidin and anti-cortactin, and analyzed by confocal laser scanning microscopy. (A and D) F-actin, (B and E) cortactin and (C and F) merged images. The merged images were shown. (A) Invadopodia were observed in the YTS control cell as F-actin puncta. (A-C) Several typical invadopodia are indicated by white arrows. Yellow indicates co-localization in the merged images. (C) Cortactin and F-actin localized at invadopodia (bar, $20 \mu \mathrm{m}$ ).

siRNA as a control (designated YTS control) was also prepared. Western blotting revealed that cortactin expression was reduced in two of the knockdown cell lines (designated cortKD-1 and -2) compared with parent YTS-1 and YTS controls (Fig. 1A). However, there was no significant difference in the growth rate between these cell lines (Fig. 1B). The results from the assays using cortKD-1 are shown and the two knockdown cell lines yielded almost identical results in all assays.

Reduction of invadopodia formation in cortactin knockdown bladder cancer cells. Cortactin promotes invadopodia formation and maturation in a number of cancer cells, and is also an invadopodium marker (8-10). Cells were double-stained with phallodin and anti-cortactin monoclonal antibody. Invadopodia in YTS control cells were visualized by Alexa Fluor 568-labeled phalloidin staining as F-actin-rich puncta (Fig. 2A). Several typical invadopodia were indicated, as shown in Fig. 2A-C. A portion of cortactin staining also exhibited a punctate pattern (Fig. 2B) and co-localization of cortactin with F-actin puncta indicated that the puncta were invadopodia (Fig. 2C). In cortKD-1 cells, cortactin knockdown resulted in a marked cell-morphological change to a round shape, and no invadopodia were observed (Fig. 2D-F). These results indicate that invadopodia formation is impaired in cortactin knockdown bladder cancer cells.

Reduced secretion of metalloproteinase in cortactin knockdown cells. To examine if cortactin knockdown affects invadopodia functions, the cells were assayed for the secretion of matrix metalloproteinase (MMP)-2, one of the most important functions of invadopodia. Gelatin zymography revealed that large amounts of MMP-2 were secreted by YTS-1 parent cells and YTS control cells (Fig. 3, lanes 1 and 2). By contrast, the secretion of MMP-2 by cortactin knockdown was markedly lower than that of YTS control cells (Fig. 3, lane 3), indicating that the ability to secrete MMP-2 was markedly reduced in cortactin knockdown cells.

In vitro invasion capacity of cortactin knockdown cells. Cortactin knockdown was found to impair invadopodia formation and secretion of MMP-2 (Figs. 2 and 3). To examine whether defective formation of invadopodia affects the invasion capacity of invasive cancer cells, a Matrigel invasion 


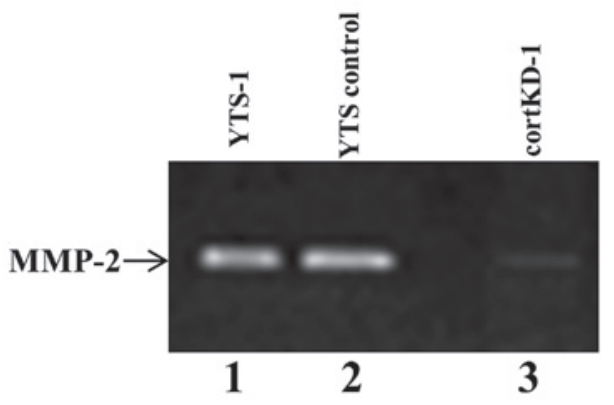

Figure 3. Reduced secretion of MMP-2 in cortactin knockdown bladder cancer cells. Conditioned media from bladder cancer cells were collected and assayed for gelatinase activity by zymography. A representative zymogram from $24 \mathrm{~h}$ conditioned medium of YTS-1 parent, YTS control and cortKD-1 cells is shown. The bands appear as clear bands against a dark background. MMP, matrix metalloproteinase; cort, cortactin; KD, knockdown.
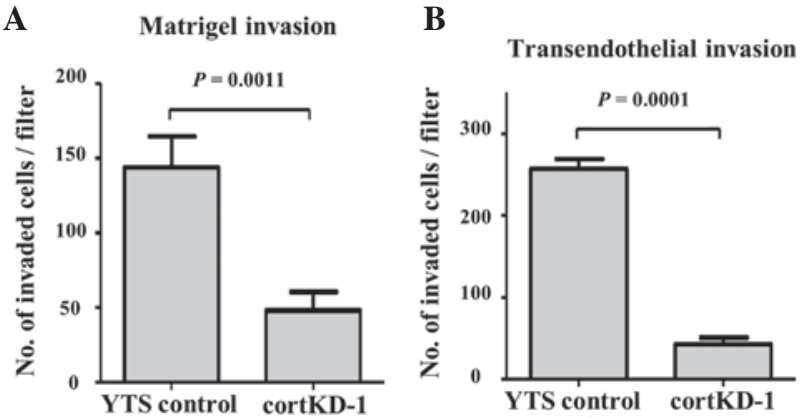

Figure 4. In vitro invasion capacity of cortactin knockdown cells (A) Matrigel invasion capacity of bladder cancer cells was measured by a conventional Matrigel invasion assay system using a Boyden chamber. (B) Transendothelial invasion capacity of bladder cancer cells. Invasion through the endothelial cells of human lung microvessels was assayed. Data represent the mean \pm standard deviation of triplicate measurements.

assay was performed. cortKD-1 cells exhibited a significantly lower invasion capacity through the Matrigel matrix (Fig. 4A). This result, taken together with Fig. 3, suggests that impaired invadopodia have a reduced capacity to degrade and migrate through the ECM.

In order for bladder cancer cells to metastasize in the lung, the cancer cells need to exit the circulation by migrating through an endothelial monolayer of the lung microvasculature. To determine the ability of cancer cells to invade and migrate through a cellular endothelial barrier, the transendothelial invasion capacity was measured. A transendothelial invasion assay was performed, as previously described (11), in which cortKD-1 cells exhibited a significantly lower transendothelial invasion capacity through the endothelial monolayer of the lung microvascular vessels (Fig. 4B). These results indicate that defective invadopodia formation by cortactin knockdown reduces the invasion capacity through a Matrigel matrix and the endothelial monolayer.

Tumor formation by cortactin knockdown cells. To evaluate the role of invadopodia in cancer cell extravasation for metastasis, the YTS control and cortKD-1 cells were subjected to a lung metastasis assay, as this type of assay closely mimics hematogenous metastasis $(7,12)$. Three weeks after cancer cell

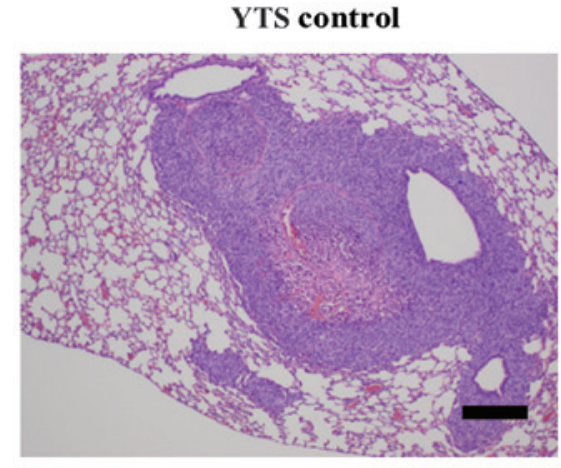

cortKD-1

B

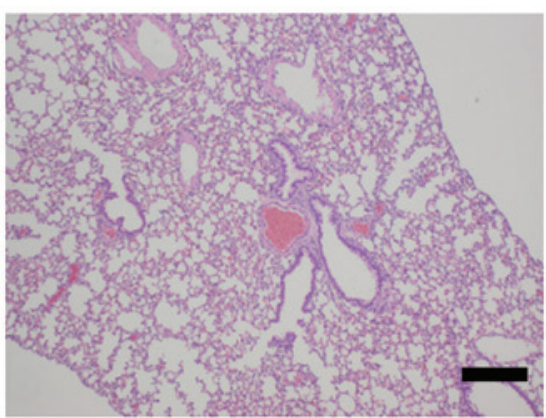

$\mathbf{C}$

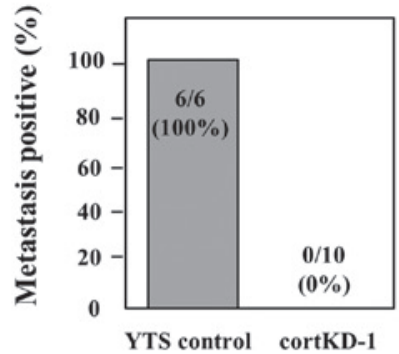

D

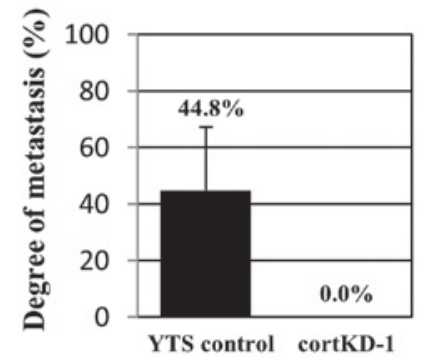

Figure 5. Lung metastasis capacity of bladder cancer cells. Lung metastasis of mice injected with (A) YTS control cells and (B) cortKD-1 cells was examined histologically. The lung sections were hematoxylin and eosin stained (bar, $0.2 \mathrm{~mm}$ ). (C) Comparison of metastasis frequency between YTS control and corKD-1 cells. (D) Comparison of the degree of metastasis between YTS control and corKD-1 cells. The degree of metastasis was evaluated by the proportion (\%) of the tumor area occupying the total lung section area. Cort, cortactin; KD, knockdown.

injection into mice, the lungs were harvested and lung sections were examined for tumorous regions. A large tumor area was observed on the lung section three weeks after YTS control cells were injected (Fig. 5A). However, no tumor area was observed on the lung section from the mice injected with cortKD-1 cells (Fig. 5B). When YTS control cells were injected, all the mice exhibited lung metastases $(n=6)$. By contrast, none of the mice injected with cortKD-1 cells exhibited detectable metastases $(n=10)$ (Fig. 5C). The degree of metastasis of YTS control 
cells was $44.8 \%$, based on the proportion (\%) of the tumor area occupying the total lung area examined at lower magnification (x40; Fig. 5D). In order for invasive bladder cancer cells to form metastatic foci in the lung, cancer cell extravasation and proliferation in the lung are required. There was no marked difference in the growth rate between YTS control and cortKD-1 cells (Fig. 1B). However, cortKD-1 cells exhibited a markedly lower degree of metastasis compared with YTS control cells (Fig. 5). These results, taken together with the lower transendothealial invasion capacity of cortKD-1 cells (Fig. 4B), suggests that the lower degree of lung metastasis is due to reduced extravasation in the lung tissues.

\section{Discussion}

Tyrosine phosphorylation of cortactin by non-receptor type tyrosine kinases, including $\mathrm{Src}$ and Arg, is a key regulatory step for invadopodia formation $(10,13,14)$. It has been demonstrated that cortactin regulates invadopodia formation in several types of cancer cell. Cortactin is also known to regulate MMP secretion and matrix degradation, contributing to the invasive character of cancer cells $(15,16)$. The present study demonstrated that cortactin plays a critical role in invadopodia formation and MMP-2 secretion in invasive bladder cancer cells (Figs. 2 and 3). Invadopodia formation regulated by cortactin was also shown to be necessary for matrix degradation and invasion by invasive bladder cancer cells (Fig. 4A).

Cancer cell extravasation is a decisive process for metastasis whereby cancer cells exit the blood vessels to cross endothelia and invade secondary organ tissues. Gligorijevi et al previously demonstrated that invadopodia formation is involved in cancer cell intravasation, using an in vivo system (17). This study knocked down the expression of neural Wiskott-Aldrich syndrome protein (N-WASP) in MTLn3 rat mammary gland adenocarcinoma cells. The N-WASP knockdown MTLn3 cells demonstrated a markedly decreased invadopodia formation, as N-WASP is an essential component of invadopodia. When the N-WASP knockdown cells were implanted into SCID mice, in vivo invadopodia formation and matrix degradation were reduced in the area of intravasation. The present study also demonstrated that invadopodia formation is involved in cancer cell extravasation, using an in vivo system. The invadopodia formation in invasive bladder cancer cells was reduced by silencing cortactin, which is another essential component of invadopodia. Subsequently, cortactin knockdown cells were subjected to the in vivo tumor formation assay, which included cancer cell extravasation. The lower rate of lung metastasis of cortKD-1 cells (Fig. 5) indicates that the extravasation of cortKD-1 cells is impaired due to reduced invadopodia formation.

In order for invasive cancer cells to extravasate, the endothelial barrier surrounding blood vessels and the basement membrane must be broken down (18). In the present study, perturbing the function of cortactin by shRNA was found to reduce MMP-2 secretion, invadopodia-mediated ECM degradation, transendothelial invasion and in vivo tumor formation by invasive cancer cells. These results indicate that cortactin-mediated invadopodia formation is required for the invasion and extravasation process of bladder cancer metastasis. In conclusion, the potential of cortactin as a target for anti-invasion and anti-extravasation therapeutics should be investigated further.

\section{Acknowledgements}

This study was supported by the grants-in-aid for Scientific Research from the Japanese Society for the Promotion of Science (nos. 22570131 and B22390301), the Ministry of Education, Culture, Sports, Science and Technology of Japan (nos. 21791483 and 21791484) and Japan Science and Technology Agency (CREST).

\section{References}

1. Steeg PS: Tumor metastasis: mechanistic insights and clinical challenges. Nat Med 12: 895-904, 2006.

2. Caldieri G, Ayala I, Attanasio F and Buccione R: Cell and molecular biology of invadopodia. Int Rev Cell Mol Biol 275: $1-34,2009$.

3. Linder S, Wiesner C and Himmel M: Degrading devices: invadosomes in proteolytic cell invasion. Annu Rev Cell Dev Biol 27: 185-211, 2011.

4. Murphy DA and Courtneidge SA: The 'ins' and 'outs' of podosomes and invadopodia: characteristics, formation and function. Nat Rev Mol Cell Biol 12: 413-426, 2011

5. Yamaguchi H, Lorenz M, Kempiak S, et al: Molecular mechanisms of invadopodium formation: the role of the N-WASP-Arp2/3 complex pathway and cofilin. J Cell Biol 168: 441-452, 2005.

6. Smith SC, Nicholson B, Nitz M, et al: Profiling bladder cancer organ site-specific metastasis identifies LAMC2 as a novel biomarker of hematogenous dissemination. Am J Pathol 174: 371-379, 2009.

7. Tsuboi S, Sutoh M, Hatakeyama S, et al: A novel strategy for evasion of NK cell immunity by tumours expressing core2 O-glycans. EMBO J 30: 3173-3185, 2011.

8. Artym VV, Zhang Y, Seillier-Moiseiwitsch F, Yamada KM and Mueller SC: Dynamic interactions of cortactin and membrane type 1 matrix metalloproteinase at invadopodia: defining the stages of invadopodia formation and function. Cancer Res 66 : 3034-3043, 2006.

9. Ayala I, Baldassarre M, Giacchetti G, et al: Multiple regulatory inputs converge on cortactin to control invadopodia biogenesis and extracellular matrix degradation. J Cell Sci 121: 369-378, 2008.

10. Oser M, Yamaguchi H, Mader CC, et al: Cortactin regulates cofilin and N-WASp activities to control the stages of invadopodium assembly and maturation. J Cell Biol 186: 571-587, 2009.

11. Sugiyama N, Yoneyama MS, Hatakeyama $\mathrm{S}$, et al: In vivo selection of high-metastatic subline of bladder cancer cell and its characterization. Oncol Res 20: 289-295, 2013.

12. Hatakeyama S, Yamamoto $\mathrm{H}$ and Ohyama C: Tumor formation assays. Methods Enzymol 479: 397-411, 2010.

13. Mader CC, Oser M, Magalhaes MA, et al: An EGFR-Src-Arg-cortactin pathway mediates functional maturation of invadopodia and breast cancer cell invasion. Cancer Res 71: 1730-1741, 2011.

14. Oser M, Mader CC, Gil-Henn H, et al: Specific tyrosine phosphorylation sites on cortactin regulate Nck1-dependent actin polymerization in invadopodia. J Cell Sci 123: 3662-3673, 2010.

15. Clark ES and Weaver AM: A new role for cortactin in invadopodia: regulation of protease secretion. Eur J Cell Biol 87: 581-590, 2008

16. Kirkbride KC, Sung BH, Sinha S and Weaver AM: Cortactin: a multifunctional regulator of cellular invasiveness. Cell Adh Migr 5: 187-198, 2011.

17. Gligorijevic B, Wyckoff J, Yamaguchi H, Wang Y, Roussos ET and Condeelis J: N-WASP-mediated invadopodium formation is involved in intravasation and lung metastasis of mammary tumors. J Cell Sci 125: 724-734, 2012.

18. Al-Mehdi AB, Tozawa K, Fisher AB, Shientag L, Lee A and Muschel RJ: Intravascular origin of metastasis from the proliferation of endothelium-attached tumor cells: a new model for metastasis. Nat Med 6: 100-102, 2000. 